\title{
Intraocular Argon Laser Photocoagulation
}

\author{
R. W. ACHESON, M. CAPON, R. J. COOLING, P. K. LEAVER, J. MARSHALL and \\ D. McLEOD
}

London

\begin{abstract}
Summary
Intraoperative photocoagulation using an endolaser delivery system is an important recent development in the field of vitreous microsurgery. Endolaser photocoagulation is indicated in a wide variety of vitreoretinal disorders and offers important advantages over previous techniques of endophotocoagulation using xenon energy. We review our experiences of argon laser endophotocoagulation to identify the role of this technique, the potential hazards and stringent precautions required for theatre personnel.
\end{abstract}

A delivery system for the intraocular application of light during closed microsurgery was first described by Charles in $1979^{1}$. Employing a portable xenon arc energy source, this technique of endophotocoagulation provided an effective method of non-contact intraocular tissue coagulation and an important new capability in vitreoretinal surgery. However, from theoretical considerations and extended clinical use of xenon endophotocoagulation, a number of inherent limitations were identified, which encouraged the development of laser endophotocoagulation systems. With advances in laser technology and fibre optics, various systems have since been devised ${ }^{2,3,4}$ and recently several systems have become commercially available.

In this presentation we review our early experiences of argon laser endophotocoagulation and briefly consider delivery systems currently available. We also describe the findings of an experimental study to determine the effects of argon endolaser photocoagulation on the retina of the monkey eye.

\section{Laser Sources and Instrumentation}

Currently there are two alternative systems for the delivery of argon or krypton laser light for endophotocoagulation. An air or water cooled laser unit can be located remotely in an area adjacent to the operating theatre, a fibre optic cable conveying laser light to a control console to which a fibre optic endolaser probe is connected (Fig. 1a). A central laser generator can therefore supply a number of operating theatres as well as a slit-lamp delivery system for out-patient use, although not simultaneously. Installation of such a system is a major and expensive undertaking and requires an area conveniently located to the operating theatre suite at a distance determined both by the length of the fibre optic and by the number of connecting points along the fibre pathway.

A preferred system uses a portable air or water cooled laser stationed in the operating theatre, the endolaser probe being directly coupled by a fibre optic line (Fig. 1b). With present commercial systems, a single phase $30 \mathrm{amp}$ electricity power supply is required. The air cooled units are smaller and lighter than their water cooled counterparts but emit considerable heat. An efficient air conditioning system in the operating theatre is therefore desirable.

Correspondence to: R. J. Cooling, F.R.C.S. Moorfields Eye Hospital, City Road, London ECIV 2PD.

Surgical Vitreoretinal Unit, Moorfields Eye Hospital, London and The Institute of Ophthalmology, London. 


\section{LASER GENERATOR UNIT-Remotely located}

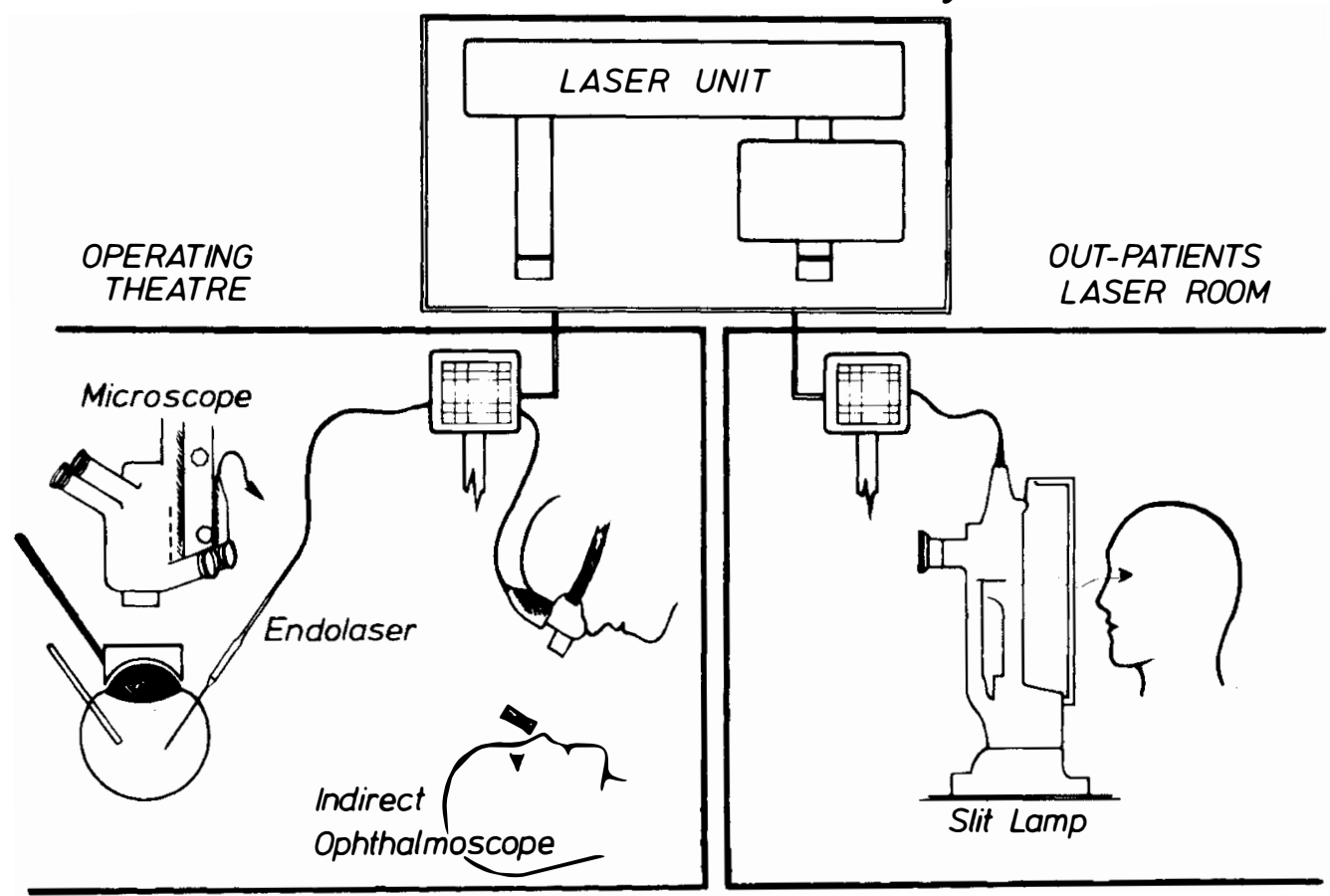

Fig. 1a. System using remotely located laser source for intraoperative and outpatients use.

\section{PORTABLE LASER GENERATOR UNIT}

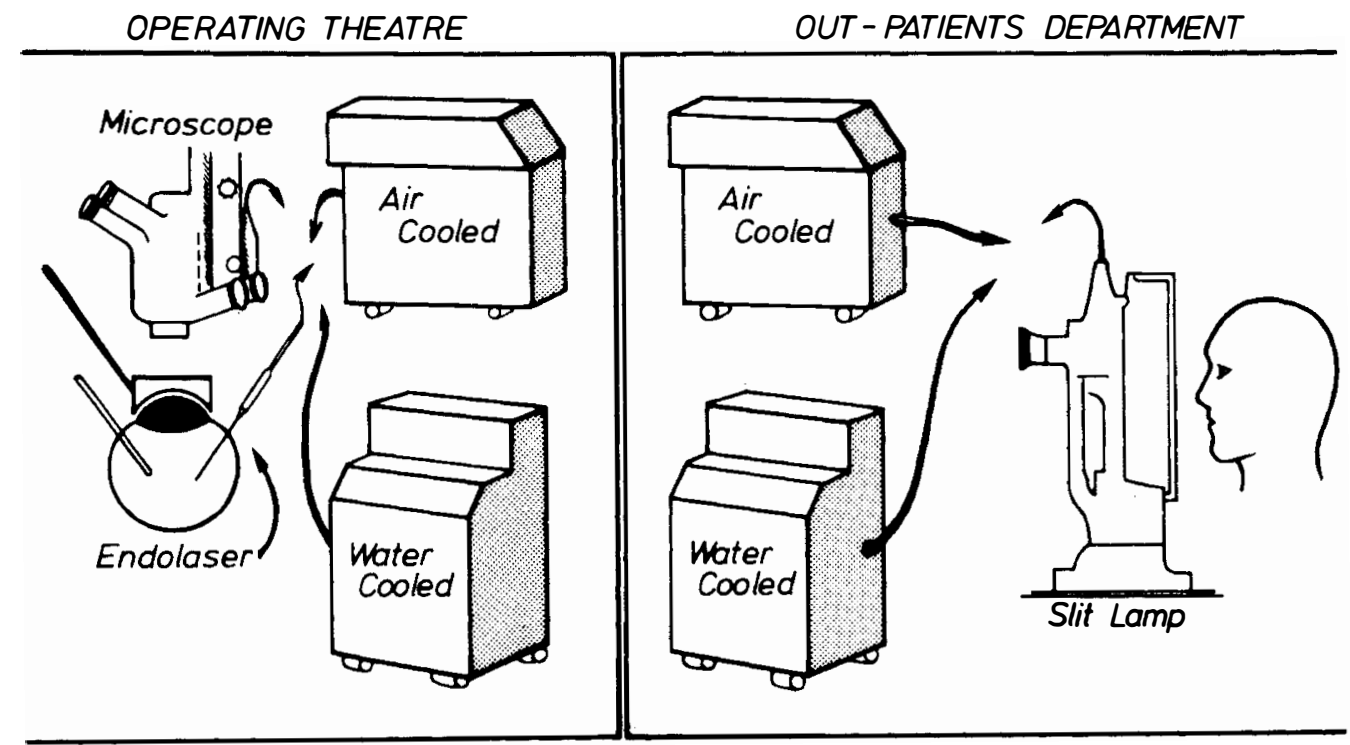

Fig. 1b. Portable laser unit for intraoperative delivery or conventional slit-lamp delivery. 
Endolaser probes designed to interface with closed microsurgical instrumentation are available in 19 and 20 gauge diameters. The fibre optic line to the control console or portable laser unit is approximately 2 metres in length.

Although observation of intraocular photocoagulation can be achieved by indirect ophthalmoscopy, the operating microscope is routinely used in conjunction with fibre optic endoillumination and a standard irrigating fundus contact lens. Focussing for photocoagulation of a gas-filled phakic eye requires a biconcave ( -90 dioptres) contact lens. ${ }^{5}$ For peripheral retinal treatment, a rotatable prism contact lens ${ }^{6}$ or Rodenstock panfundoscope lens ${ }^{7}$ may be used and in the aphakic eye coaxial illumination is combined with scleral indentation.

Protection for the operator and observers against exposure to reflected laser light is provided by an appropriate filter introduced into the viewing system of the operating microscope or by an electronically controlled shutter introduced below the objective lens. Observation by indirect ophthalmoscopy requires the use of a filter in the plane of the assistant's viewing mirror or the eye pieces of the ophthalmoscope with the assistant and other personnel wearing protective spectacles or goggles.

\section{Clinical Endolaser Photocoagulation}

During a 10 month period from the installation of an argon endolaser system in March 1985 , a total of 131 patients underwent endolaser photocoagulation at Moorfields Eye Hospital. Following vitrectomy, all patients were treated with mixed blue/green argon laser light from a portable $3.5 \mathrm{~W}$ air cooled unit (HGM model 5) delivered through a 19 gauge fibre optic probe. Endolaser application was carried out in Hartmann's solution, gas (air/sulphahexafluoride) or silicone oil and observed through the operating microscope in combination with fibre optic endoillumination and fundus contact lens where appropriate. The usual power setting was 0.3 watts and did not exceed 0.6 watts. The size of the burn was determined by the distance of the probe tip from the retinal surface, smaller burns being produced by advancing towards the retina from reduced divergence of the laser beam. All participating surgeons preferred the probe tip to be located at approximately $3 \mathrm{~mm}$ from the retinal surface and a continuous exposure setting operated by the foot pedal switch terminated when a pale white reaction was induced in the underlying retina.

An adequate reaction sometimes proved difficult to achieve in areas of severe intraretinal oedema and laser therapy occasionally had to be deferred until the post operative period depending upon the clarity of the ocular media. A further operative difficulty was the frequent formation of small gas bubbles within the probe tip interfering with the transmission of laser energy to the retina and thought to be related to multiple re-use of the fibre optic laser probe and a localised heating effect upon air trapped within the probe. This phenomenon posed the danger of a compensatory increased power setting on the part of an unsuspecting surgeon and, with subsequent dispersal of the bubbles, over treatment of the retina with possible choroidal disruption.

Protection from reflected laser light was provided by a manually operated filter incorporated within the microscope viewing system. Introduction of the filter hampered visualisation of the aiming beam and in addition, the intensity of laser treatment appeared reduced with the tendency towards over treatment. This problem could be eliminated in the future by the use of holographic filters. A theatre technician was always at hand to operate the controls in accordance with local laser safety rules, the laser beam being activated only when the endolaser probe was located within the eye and upon the instructions of the operating surgeon. By defining the laser cavity to be within the globe, the need for other theatre personnel to wear protective filter devices was eliminated.

\section{Indications (Table I)}

In order to determine the principal uses, treatment parameters and complications of laser endophotocoagulation, we reviewed the clinical records of 131 patients undergoing treatment, 12 patients being excluded because of insufficient data. 
Table I

\begin{tabular}{lr}
\hline \multicolumn{2}{c}{ Endolaser photocoagulation-indications } \\
\hline Proliferative Diabetic Retinopathy & 59 \\
Macular and Posterior Retinal Breaks & 37 \\
Proliferative Vitreoretinopathy & 13 \\
Non-diabetic vasoproliferative retinopathy & 8 \\
Others & 2 \\
Total & 119 \\
\hline
\end{tabular}

Our principal indication for intraoperative photocoagulation was treatment of ischaemic retina in patients with advanced diabetic eye disease, including macular traction retinal detachment, non-resolving vitreous haemorrhage and combined traction and rhegmatogenous retinal detachment. In those eyes which had not undergone previous retinal photocoagulation, 700-1,200 burns were applied in a scatter pan-retinal distribution whereas in other patients treatment was confined to previously untreated areas to achieve a complete pan-retinal ablation. Direct treatment was only occasionally applied to areas of flat retinal neovascularisation since removal of attached vitreous cortex was one of the essential objectives of microsurgery, whereas coagulation of pre-retinal neovascularisation or disc vessels was achieved by alternative methods, e.g. bimanual bipolar diathermy.

In a small number of cases, scatter endophotocoagulation was used to combat the effects of intraretinal ischaemia resulting from non-diabetic vasoproliferative retinopathy, e.g. branch retinal vein occlusion, Eales' disease, Behçet's and sickle cell disease. In 30 per cent of the eyes, endophotocoagulation was undertaken for the treatment of macular or other posterior retinal breaks, either preexisting or iatrogenic. Such breaks were associated with retinal detachments following previous trauma, high myopia, as a complication of epiretinal dissection and other miscellaneous causes, e.g. Stickler's syndrome, colobomatous malformations. Following vitrectomy and retinal re-attachment including fluid/gas or silicone oil exchange, endolaser photocoagulation was applied in two or three rows of confluent burns to surround the retinal break with the exception of macular holes around which a single row of burns was applied.
Endolaser photocoagulation was employed to seal small peripheral retinal breaks in retinal detachments complicated by proliferative vitreoretinopathy, and to the posterior flap of spontaneous giant retinal tears and those created by deliberate circumferential relieving retinotomies. Following hydraulic or pneumatic retinal re-attachment, laser photocoagulation was applied to surround all retinal breaks and in selected cases in a more widespread scatter pattern to the post-equatorial retina.

Serious complications arising in the course of treatment were noted in four cases including a choroidal haemorrhage, an extensive serous choroidal effusion and a burn through to the sclera. In each case, the complication arose in the early phases of experience with this technique and was attributable to excessive energy levels. In a further case, multiple retinal breaks were created in atrophic retina surrounding a posterior retinal break in a case of morning glory syndrome. No adverse effects were identified from the uptake of stray laser light posterior to indented peripheral retina during treatment or from the total internal reflection of light energy from the meniscus of a liquid silicone bubble.

\section{Comment}

The original concept of endophotocoagulation introduced by Charles represented a significant therapeutic advance. However, with the use of the xenon arc system, high beam divergence and energy loss from destructive light interference with increasing distances from the retina necessitated a working distance from the retinal surface of less than $1 \mathrm{~mm} .{ }^{4}$ In addition to the risk of mechanical injury, damage to the retina and the fibre optic probe can result from local heating effects unless the probe tip is immersed in an aqueous or sodium hyaluronate medium. ${ }^{8}$ In contrast, laser endophotocoagulation with the advantages of coherence and high energy density with minimal heat dissipation enables moderate sized burns to be produced at greater distances from the retina and for the energy to be delivered through an aqueous, gas or liquid silicone medium. Furthermore, a more rapid firing sequence can be achieved which together with reduced excursion of the 


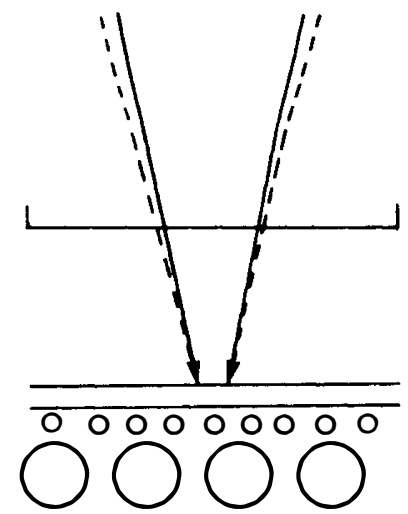

(a)

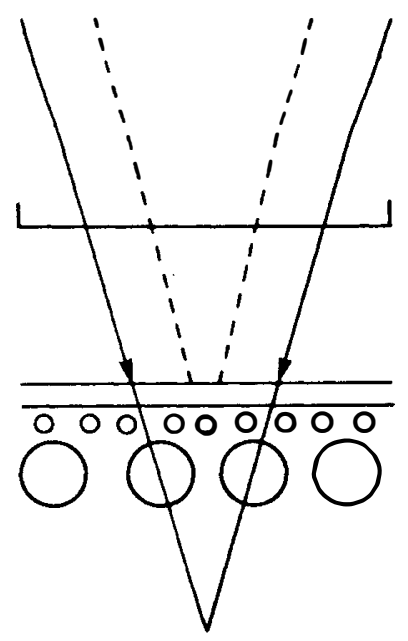

FIG 2

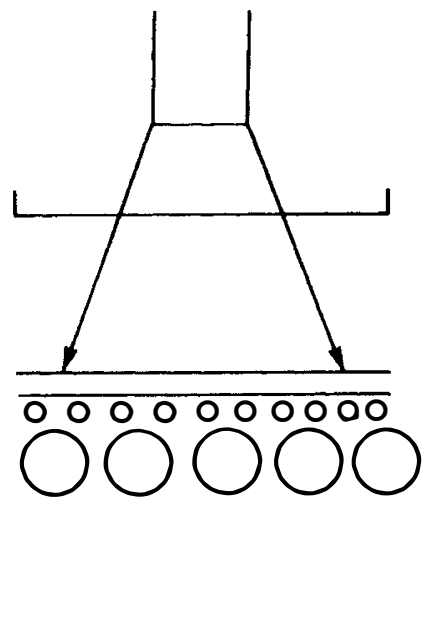

ILM
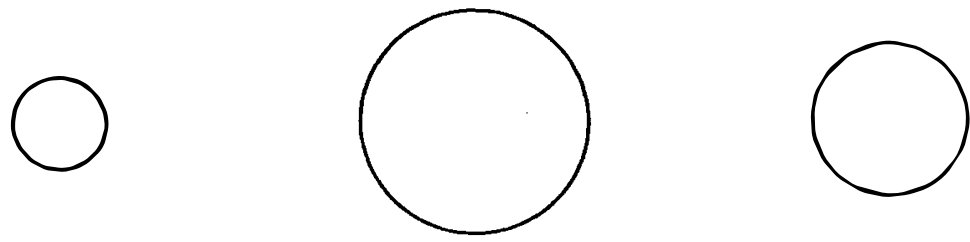

RPE
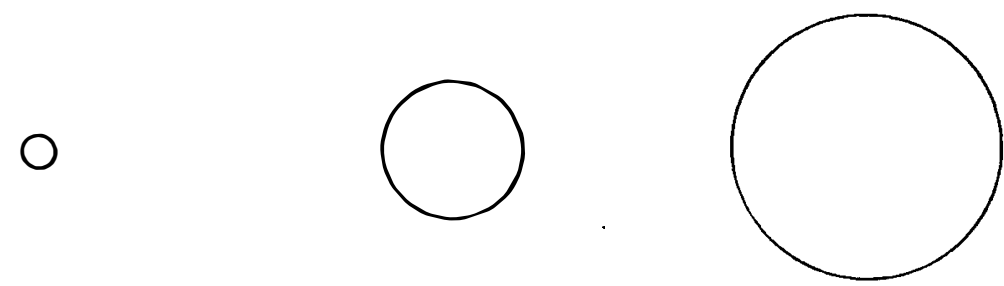

(b)

500

Endo

Fig. 2. Diagram summarising the major differences between photocoagulation using conventional lasers (left and centre) and endolasers (right). The beam parameters are shown in elevation in the upper picture and in plan in the lower picture. In most conventional photocoagulators small image sizes are achieved by making the treatment beam and aiming beam parfocal. In such a situation a convergent beam is imaged on the retinal pigment epithelium (RPE) and thus the highest irradiances occur at this plane. Because the beam is highly focused and image sizes relatively small there is also a relatively high irradiance at the level of the inner limiting membrane (ILM). In the case of larger image sizes many manufacturers achieve this by changing the relative focus of the aiming beam and treatment beam ie. by pulling the focusing beam back up the path length of the treatment beam a larger image size can effectively be achieved. It is important to note however that in such cases the highest irradiance may be well below the focal plane of the aiming beam. In such cases the irradiance is again higher at the pigment epithelium than at the inner limiting membrane but here the highest irradiance may be within the choroid itself. In contrast with endophotocoagulation the highest irradiance is always at the level of the inner limiting membrane. The highest absorbed energy density however is always at the level of the pigment epithelium because this is where the maximum concentration of pigment per unit volume occurs. 


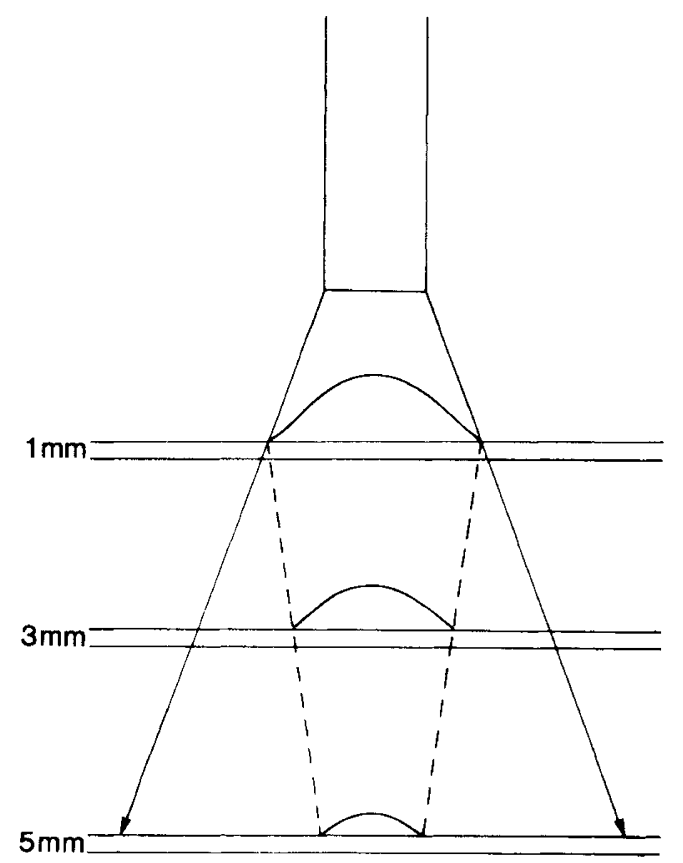

Fig. 3. Diagrammatic representation of the effect of pulling the endotip progressively further away from the retinal surface. The double lines labelled $1 \mathrm{~mm}, 3 \mathrm{~mm}$ and $5 \mathrm{~mm}$ are meant to represent the retinal pigment epithelium at the respective distances. In this case as the probe tip is withdrawn from the retinal surface, there is a progressive increase in the size of the area of pigment epithelium irradiated. Given a constant incident energy there will be a progressive decrease in irradiance. This will also reflect in a decrease in the energy absorbed per unit volume of tissue and there fore the temperature rises experienced within the target will progressively decrease. This is diagrammatically represented by the gaussian curves bordered by the dotted lines. In practice surgeons may not see this effect as they tend to increase the incident energy as they remove the tip further and further from the retinal surface.

probe tip allows extensive retinal photocoagulation to be accomplished with far greater efficiency. Since argon blue/green light is preferentially absorbed by the retinal pigment epithelium, mild endolaser photocoagulation may be limited in its effects to the outer neuroretina in contrast to xenon endophotocoagulation.

Laser endophotocoagulation may also offer important advantages compared with other methods of retinal coagulation and in particular trans-scleral cryotherapy. The latter is associated with oedema of the choroid and retina with dispersal of potentially fibroblastic cells from the retinal pigment epithelium due to destruction of the full thickness of the retina and release of significant amounts of fibronectin. Endolaser photocoagulation may possibly eliminate or reduce these effects and thereby allow extensive retinal coagulation to be carried out with reduced potential for epiretinal cellular proliferation.

The most important attribute of intraoperative photocoagulation is the ability to undertake or complete pan-retinal photocoagulation of ischaemic retina in advanced diabetic eye disease so reducing the stimulus to vasoproliferation and the risks of post-operative re-bleeding and rubeosis iridis ${ }^{9,10}$. Frequently, post-operative scatter photocoagulation is precluded by the presence of residual vitreous opacity or further haemorrhage in the early post-operative period.

Laser endophotocoagulation provides an extremely precise technique of retinal coagulation for the treatment of posterior retinal breaks with less risk of inadvertent damage to the fovea or optic nerve. The management of retinal detachment associated with macular breaks, in particular the need for macular coagulation, remains controversial but a single row of endolaser photocoagulation to the immediate perifoveal area does not appear to compromise ultimate visual recovery.

Recently, use of the endolaser probe in an extraocular location has been described for the drainage of subretinal fluid ${ }^{11}$. This allows cauterisation of the exposed choroid and controlled drainage through a small choroidal penetration without the need to penetrate the subretinal space. A perpendicular approach is required, but the more posterior drainage sites, oblique positioning of the probe with an increased power setting carries the risk of damage to the posterior retina from the transmission of energy across the vitreous cavity. The use of the endolaser probe under these circumstances obviously demands strict observation of laser safety precautions.

\section{Experimental Endolaser Photocoagulation}

Although the histopathological effects of conventional laser-induced lesions of the retina have been well documented, few publications deal with the histopathology of endolaser 


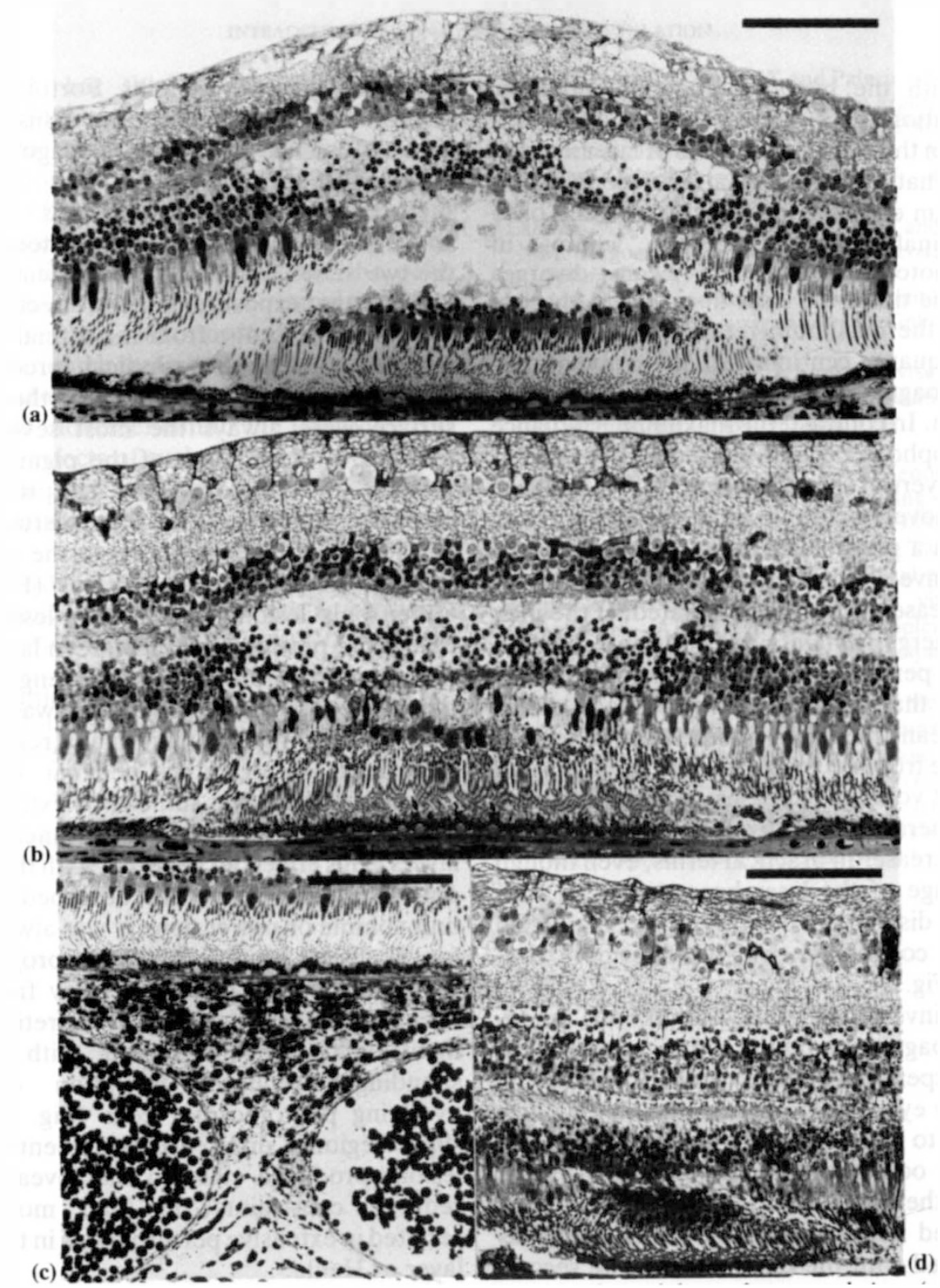

Fig. 4. Light micrographs of lesions produced in monkey retina by endolaser photocoagulation. (a) and (b) lesions produced with tip $1 \mathrm{~mm}$ from retina using blue/green (a) and green (b) respectively. Note in both cases primary damage is confined to the outer retinal layers involving the pigment epithelium and overlying photoreceptor cells. The damage to the fibre layer of Henle and outer plexiform layer in both cases derives from fluid disturbances or oedema. In (a) the volume of oedema has been sufficient to permeate the inner nuclear layers and also to cause some destruction in the inner plexiform layer. These do not however represent damage caused by direct absorption in these layers.

(c) Light micrograph of area of choroid beneath lesions seen in (a) above. Note the closure of the choriocapillaris and the relative disorganisation in the adjacent choroidal layers caused by dissipation of heat induced by the absorption of blue/green laser light in the choroid. Note also the adhesion of a large number of white blood cells to the vessel walls of the two major choroidal vessels shown.

(d) Light micrographs showing an endolaser lesion induced by a green only exposure $3 \mathrm{~mm}$ from the retinal surface and at the same energy level as $(a)$ and $(b)$. It is important to note in this picture that the disturbances in the outer retina are far less severe than those seen in (b) above. Note also that at this level of irradiance the secondary disturbances due to oedema are minimal.

The bar markers are $100 \mathrm{um}$. 
deal with the histopathology of endolaser coagulation. A fundamental difference between these two techniques of laser irradiation is that in conventional photocoagulation the beam employed is directly focussed onto the retinal pigment epithelium, whereas in endophotocoagulation the beam diverges from the tip of the laser fibre. In biophysical terms, the maximum retinal irradiance (i.e. watts/square centimetre) in conventional photocoagulation occurs at the pigment epithelium. In contrast, the maximum irradiance of endophotocoagulation occurs at the innermost layers of the retina (Fig. 2). As the fibre tip is moved further from the retinal surface, there is a progressive decrease in irradiance (from inverse square law considerations) and an increase of the area irradiated. If the incident energy is maintained constant, then the energy per unit area will be reduced. This implies that whilst the optical image of the laser beam increases in size with increasing distance from the retina, the energy deposited per unit volume decreases. The thermal profiles generated by such energy deposition will also decrease. In practical terms, even though the image of the laser beam increases with greater distances from the retina, the actual area of coagulation will become more confined (Fig. 3).

To investigate the effects of endophotocoagulation on the retina, a series of in vivo experiments were carried out in the monkey eye. These experiments were undertaken to determine whether significant damage occurred to the inner retinal layers due to their absorption of laser energy from increased irradiance at the inner retina as compared with the outer retina. The second series of experiments were carried out to document the effects of positioning the tip of the laser probe progressively further from the retinal surface. In order to ascertain the effects of absorption in the inner and outer retinal layers, two different laser wavelengths were used, namely the green only and mixed blue/green options.

Two parallel series of exposures of 200 millisecond duration and 100 milliwatts were created at distances of 1,3 and $5 \mathrm{~mm}$ from the surface of the retina respectively. The exposures were carried out using green only and blue/green wavelengths. Fortuitously, a further variable introduced into this experimental sequence was the photocoagulation of different areas of the retina.

Histopathological preparations of the above series of lesions demonstrated that in the two series with constant incident energy and constant exposure time the effect of withdrawal of the probe from the retinal surface correlated with the physical predictions. Lesions produced at $1 \mathrm{~mm}$ from the retinal surface were always the most severe and resulted in coagulation of the pigment epithelium and photoreceptor cell tips with secondary influx of oedema and disruption of all the retinal layers external to the synaptic layer of the photoreceptor cells (Fig. 4a). Green only lesions were always less severe than those produced by blue/green laser light (Fig. 4b). With blue/green wavelengths, significant choroidal involvement was documented (Fig. 4c) and this usually resulted in more marked intraretinal oedema. In some cases the oedema was sufficiently extensive to cause secondary damage in the inner retinal layers. This effect did not occur with the green only exposures where the absorbed power/ unit volume or power density was always less for a given irradiance. With progressive movement of the probe tip away from the retina the extent of damage at the retinal pigment epithelium decreased with correspondingly reduced disruption of the overlying photoreceptor cells (Fig. 4d). A slight regional difference was identified in lesions produced close to the fovea where reduced constraints on fluid movement resulted in extensive perturbations in the fibre layer of Henle.

The findings of our experimental studies clearly show that for a given power setting as the endolaser probe tip is moved further away from the retina, the irradiance decreases and the thermal disturbances resulting from the reducing energy absorbed within the pigment epithelium causes correspondingly reduced damage. They also demonstrated that no damage was caused to the inner layers of the retina by primary absorption of laser energy with either the green only or blue/green options. Damage was occasionally seen in the inner retinal layers following blue/green 
exposures, but this always resulted from secondary effects of fluid movements within the retina. In terms of the clinical uses of endophotocoagulation, it would therefore seem that the optimal working distance from the surface of the retina is at or in excess of $3 \mathrm{~mm}$. If this distance is reduced, the peak irradiances achieved at the retinal pigment epithelium and innermost layers of the choroid are such that a massive disturbance of the blood-retinal barrier occurs with significant fluid movements into the overlying retina. The effects of such fluid disturbances on any subsequent adhesion between the retina and the underlying tissues are unknown.

We wish to thank Evelyn O'Keefe for preparing the illustrations, June Krafft for the histopathological preparations and Heather Lucas for secretarial assistance.

Our particular thanks are due to Help the Hospitals Charitable Trust for their generous funding of the endolaser photocoagulator.

\section{References}

${ }_{1}$ Charles S: Endophotocoagulation. Ophthalmol. Times, 1979; 4: 68-9.
2 Peyman GA, Grisolano, JK and Palacio MN: Intraocular photocoagulation with the argon-krypton laser. Arch. Ophthalmol. 1980; 98: 2062-4.

${ }^{3}$ Fleischman, JA, Swartz M and Dixon JA: Argon laser endophotocoagulation. Arch. Ophthalmol., 1981; 99: 1610-2.

${ }^{4}$ Landers MB, Trese MT, Stefansson E. and Bessler M: Argon laser intraocular photocoagulation. Ophthalmology, 1982; 89: 785-8.

${ }^{5}$ Landers MB, Stefansson E. and Wolbarsht ML: The optics of vitreous surgery. Am. J. Ophthalmol., 1981; 91: 611-4.

${ }^{6}$ Rinkoff JS and Landers MB: Improved visualisation of the retina during endophotocoagulation. Am. J. Ophthalmol., 1984; 98: 805.

${ }^{7}$ Spitznas. Vail Meeting, 1986.

${ }^{8}$ Topping TM and Williams GA: Xenon endophotocoagulation under air using sodium hyaluronate. Am. J. Ophthalmol., 1984, 97: 785.

${ }^{9}$ Fleischman, JA, Swartz M. and Aaberg TM: Argon laser endophotocoagulation. Medical trials suggest decreased incidence of post vitrectomy rubeosis. ARVO abstracts. Supplement to Invest. Ophthalmol. Vis. Sci. Philadelphia, 1984, 258.

${ }^{10}$ Ficker L, Passani F., Leaver PK and McLeod D: Xenon are endophotocoagulation during vitrectomy for diabetic vitreous haemorrhage. Graefe's Arch. Clin. Exp. Ophthalmol., 1986 (in press).

${ }^{11}$ Bovino JA, Marcus DF, and Nelsen PT: Argon laser choroidotomy for drainage of subretinal fluid. Arch. Ophthalmol., 1985; 103: 443-4. 